\title{
Electrospun Nanoporous Poly(butylenes succinate-co-bytylene terephthalate) Nonwoven Mats
}

\author{
Liang Wang, ${ }^{1}$ Yang-Shuai Liu, ${ }^{1}$ Lu-Feng Mo, ${ }^{1}$ Fu-Juan Liu, ${ }^{2}$ and Lan $\mathrm{Xu}^{2}$ \\ ${ }^{1}$ College of Textile, Donghua University, Shanghai 201620, China \\ ${ }^{2}$ National Engineering Laboratory for Modern Silk, College of Textile and Engineering, Soochow University, 199 Ren-ai Road, \\ Suzhou 215123, China
}

Correspondence should be addressed to Liang Wang, liangwang1988@gmail.com

Received 8 June 2011; Accepted 29 August 2011

Academic Editor: Peter Majewski

Copyright (c) 2011 Liang Wang et al. This is an open access article distributed under the Creative Commons Attribution License, which permits unrestricted use, distribution, and reproduction in any medium, provided the original work is properly cited.

A traditional Chinese drug "Yunnan Baiyao" is used as an additive in poly(butylenes succinate-co-bytylene terephthalate) (PBST) solution, which is a kind of biodegradable aliphatic-aromatic copolyesters, to produce microspheres with nanoporosity by electrospinning; the tunable size of nanoporosity can be controlled by changing the voltage applied in the electrospinning process.

\section{Introduction}

The performance and applications of nanomaterials strongly depend on their morphological and structural properties [1, 2 ]. An obvious phenomenon is the remarkably large surfaceto-volume ratio of nanomaterials, and porous nanofibers can result in extraordinary increase of the ratio [3-9], which is very much suitable for biomedical applications $[10,11]$, such as drug release. To this end, electrospinning technology is used to fabricate nanoporous materials using a Chinese traditional drugs called "Yunnan Baiyo" as an additive [7].

\section{Biocompatible Materials}

Materials for biomedical applications should have not only good mechanical properties, but also excellent biocompatibility and cytocompatibility. Therefore, biocompatible polymers are electrospun to nonwoven mats. Sometimes, surface or volume modification is needed to ensure the property of biomaterials. Biodegradable polymers including poly(lactic-co-glycolic acid) (PLGA) and PCL, and water soluble biomaterials such as poly(ethylene oxide) (PEO) and polyvinyl alcohol (PVA), can be easily electrospun into nanofibers.

In this paper, poly(butylenes succinate-co-bytylene terephthalate) (PBST), a kind of aliphatic-aromatic copolyesters, is chosen to be the base material for electrospinning.
It shares desirable biodegradability and good mechanical properties [12]. A kind of Chinese traditional drugs called "Yunnan Baiyo," which is a highly valued and important traditional Chinese drug, is used as an additive. There are a wide variety of therapeutic uses of "Yunnan Baiyo," including the promotion of blood circulation, removal of blood stasis, anti-inflammatory action, hemostasis, induction of blood clotting, relief of swelling, and alleviation of pain, it can also be used for the treatment of traumatic injury, spitting blood, hemoptysis, surgical bleeding, suppurative and pyogenic infections, soft tissue bruise, closed fracture, infective diseases on skin and so forth. It also has potential applications in wound dressings [7].

\section{Experimental}

3.1. Materials. Poly(butylenes succinate-co-bytylene terephthalate) (PBST) with a molecular weight of $20,000 \mathrm{~g} / \mathrm{mol}$ was supplied by Shanghai Institute of Organic Chemistry. PBST pellets were dissolved in a mixed solvent of isopropylalcohol and chloroform with a weight ratio $9: 1$. The solvents were bought from Shanghai Chemical Reagent Co., Ltd., China. "Yunnan Baiyao," however, a traditional Chinese drug, was produced by Yunnan Baiyao Group Co. Ltd., China. All materials were used without any further purification. 


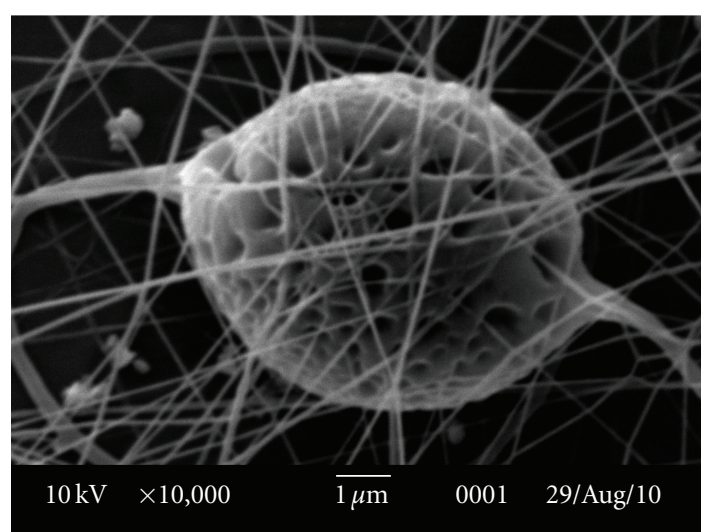

FIGURE 1: SEM image of the electrospun porous microspheres. The voltage is $20 \mathrm{KV}$, the diameter of micropores varies from $600 \mathrm{~nm}$ to $800 \mathrm{~nm}$.

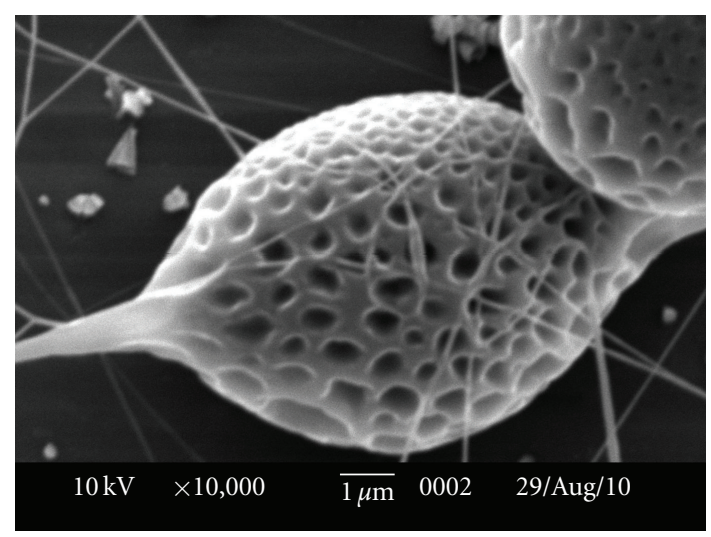

Figure 2: SEM image of the electrospun porous microspheres. The voltage is $25 \mathrm{KV}$, the diameter of micropores varies from $250 \mathrm{~nm}$ to $500 \mathrm{~nm}$.

PBST and "Yunnan Baiyao" were dissolved in the mixed solvent with the weight ratio 1:9:90 ("Yunnan Baiyao": PBST: mixed solvent).

3.2. Electrospinning Process. The electrospinning setup includes a high voltage power supply (up to $50 \mathrm{kV}$ ), a syringe, a syringe pump, a flat metal needle, and a grounded collector. The collection distance was $12 \mathrm{~cm}$, environment temperature was $27^{\circ} \mathrm{C}$, and relative humidity was $60 \%$. In the electrospinning process, a charged polymer solution is extruded toward a grounded collector plate. In sufficiently high electric field, the polymer stream is whipped around, leading to the formation of a nonwoven mat of submicrometer-diameter fibers. The diameter of the orifice is $0.7 \mathrm{~mm}$, the voltage is adjusted to $10-30 \mathrm{KV}$, and the flow rate is adjusted artificially.

\section{Results and Discussion}

The morphology of the electrospun nonwoven mats was investigated by a scanning electron microscope (SEM). The films were collected on an SEM disk and coated with gold before photographing. SEM micrographs are illustrated in Figures 1, 2, and 3 .

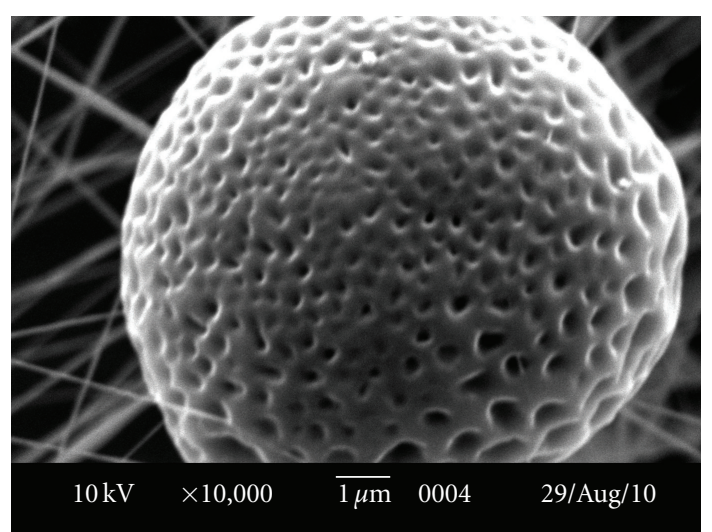

FIGURE 3: SEM image of the electrospun porous microspheres. The voltage is $35 \mathrm{KV}$, the diameter of micropores varies from $100 \mathrm{~nm}$ to $200 \mathrm{~nm}$.

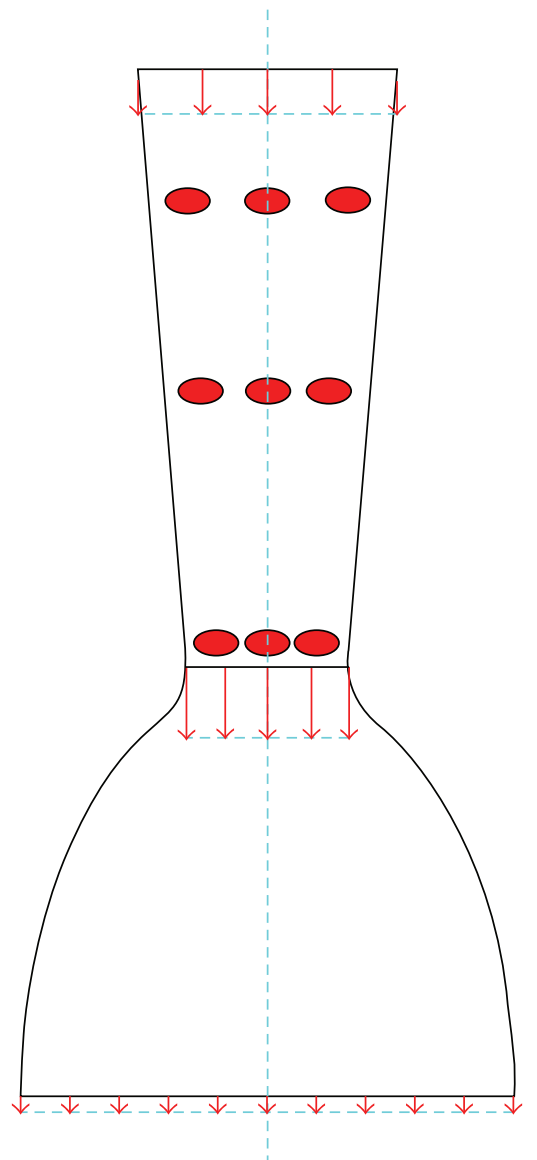

FIgURE 4: Macromolecular chains are compacted during the electrospinning.

4.1. Electrospinning Dilation. According to the continuity of the charged flow,

$$
\frac{1}{4} \pi d^{2} u \rho=Q
$$

where $d$ is the diameter of the jet, $u$ the velocity, $\rho$ the density of the solution, and $Q$ flow rate, the radius of the jet decreases with the increase of the velocity of the incompressible 


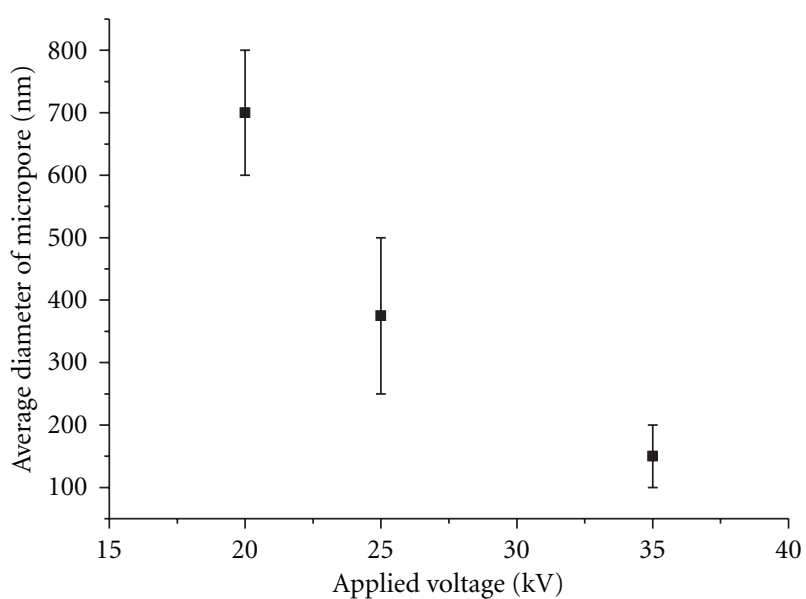

Figure 5: Relationship between the size of microspheres and the applied voltage.

charged jet. When the velocity exceed, a critical value $u_{c r}$, electrospinning dilation, see Figure 4 or [13], happens which leads to microspheres with nanoporosity as illustrated in Figure 5. It is obvious that the size of nanoporosity can be controlled by changing the applied voltage.

4.2. Nanoeffect. According to the size effect of mechanical properties, electrospun fibers on the nanoscale possess higher strength and Young modulus. Such fibers are possiblly applied in tissue engineering like connective tissue which provides structural and mechanical support for the body and works in conjunction with muscle tissue for coordinated and reflexive movements by acting as the lever arm [10].

\section{Conclusion}

In conclusion we suggested a general strategy for the synthesis of microspheres with nanoporosity by electrospinning, the porous sizes having uniform but tunable diameters can be controlled by changing the voltage applied in the electrospinning process. The flexibility and adaptation provided by the method have made the method a strong candidate for producing nanoporous materials for biomedical applications, such as tissue engineering, drug release, and the bubble electrospinning [13-16], can be used for volume production of nanoporous materials.

\section{Acknowledgment}

This work is supported by a project funded by the Priority Academic Program Development (PAPD) of Jiangsu Higher Education Institutions, National Natural Science Foundation of China under Grant nos. 10972053 and 10802021.

\section{References}

[1] L. Xu, Y. Wu, and Y. Liu, "Electrospun nanoporous materials: reality, potential and challenges," Materials Science and Technology, vol. 26, no. 11, pp. 1304-1308, 2010.
[2] J. H. He, Y. Liu, and L. Xu, "Apparatus for preparing electrospun nanofibres: a comparative review,", Materials Science and Technology, vol. 26, pp. 1275-1287, 2010.

[3] M. Kanehata, B. Ding, and S. Shiratori, "Nanoporous ultrahigh specific surface inorganic fibres," Nanotechnology, vol. 18, no. 31, Article ID 315602, 2007.

[4] Y. Miyauchi, B. Ding, and S. Shiratori, "Fabrication of a silverragwort-leaf-like super-hydrophobic micro/nanoporous fibrous mat surface by electrospinning," Nanotechnology, vol. 17, no. 20, article no. 019, pp. 5151-5156, 2006.

[5] L. Zhang and Y. L. Hsieh, "Nanoporous ultrahigh specific surface polyacrylonitrile fibres," Nanotechnology, vol. 17, no. 17, pp. 4416-4423, 2006.

[6] J. H. He, Y. Liu, L. Xu, and J. Y. Yu, "Micro sphere with nanoporosity by electrospinning," Chaos, Solitons and Fractals, vol. 32, no. 3, pp. 1096-1100, 2007.

[7] L. Xu, J. H. He, and Y. Liu, "Electrospun nanoporous spheres with Chinese drug," International Journal of Nonlinear Sciences and Numerical Simulation, vol. 8, no. 2, pp. 199-202, 2007.

[8] Y. Hong, X. Chen, X. Jing et al., "Preparation, bioactivity, and drug release of hierarchical nanoporous bioactive glass ultrathin fibers," Advanced Materials, vol. 22, no. 6, pp. 754$758,2010$.

[9] J. Y. Lin, B. Ding, J. Y. Yu, G. C. Wu, J. M. Yang, and G. Sun, "Effect of porous structure of electrospun fibers on their specific surface area: theoretical analysis and experimental verification," International Journal of Nonlinear Sciences and $\mathrm{Nu}$ merical Simulation, vol. 11, no. 7, pp. 523-527, 2010.

[10] W. J. Li, C. T. Laurencin, E. J. Caterson et al., "Electrospun nanofibrous structure: a novel scaffold for tissue engineering," Journal of Biomedical Materials Research, vol. 60, pp. 613-621, 2001.

[11] W. J. Li, K. G. Danielson, P. G. Alexander, and R. S. Tuan, "Biological response of chondrocytes cultured in three-dimensional nanofibrous poly( $\varepsilon$-caprolactone) scaffolds," Journal of Biomedical Materials Research Part A, vol. 67, no. 4, pp. 11051114, 2003.

[12] F. X. Li, X. J. Xu, Q. Li et al., “Thermal Degradation and their Kinetics of Biodegrable Poly(Butylenes Succinate-co-bytylene Terephthalate) under nitrogen and air atmosperes," Polymer Degradation and Stability, vol. 91, pp. 1685-1693, 2006.

[13] J. H. He, Y. Liu, L. F. Mo, Y. Q. Wan, and L. Xu, "Elelectrospun Nanofibres and their applications," Smithers Rapra Update, Shawbury, UK, 2008.

[14] R. R. Yang, J. H. He, J. Y. Yu et al., "Bubble-electrospinning for fabrication of nanofibers with diameter of about 20nm," International Journal of Nonlinear Sciences and Numerical Simulation, vol. 11, pp. 163-164, 2010.

[15] Q. Yang, J. H. He, and L. F. Mo, "Bubble-electrospinning for Polyacr-ylonitrile(PAN) Nanofibers," International Journal of Nonlinear Sciences and Numerical Simulation, vol. 11, pp. 165169,2010

[16] Y. Liu and J. H. He, "Bubble electrospinning for mass production of nanofibers," International Journal of Nonlinear Sciences and Numerical Simulation, vol. 8, no. 3, pp. 393-396, 2007. 

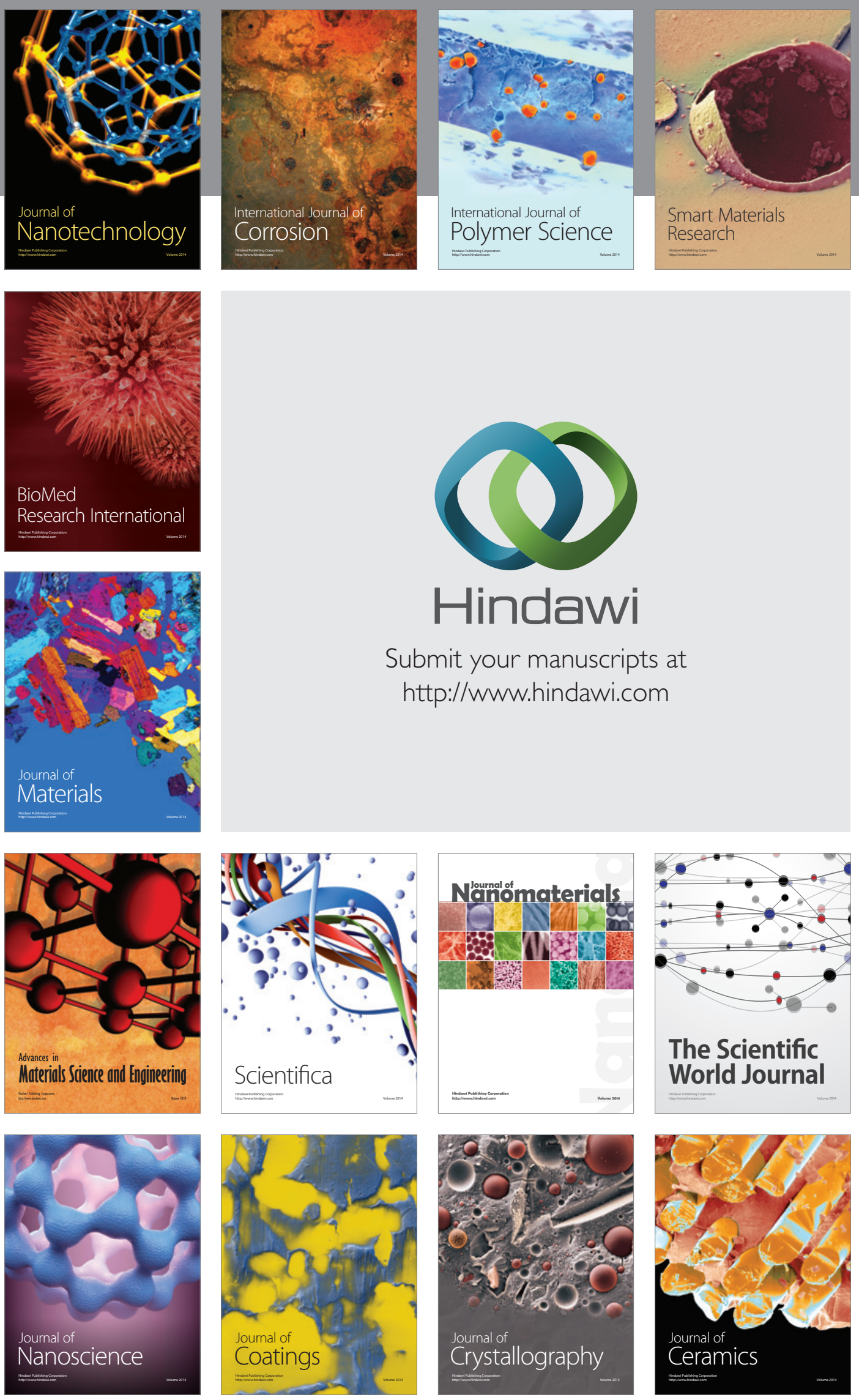

The Scientific World Journal

Submit your manuscripts at

http://www.hindawi.com

\section{World Journal}

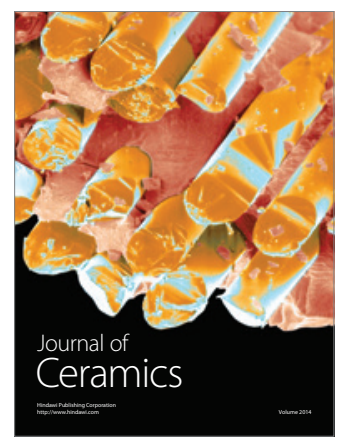

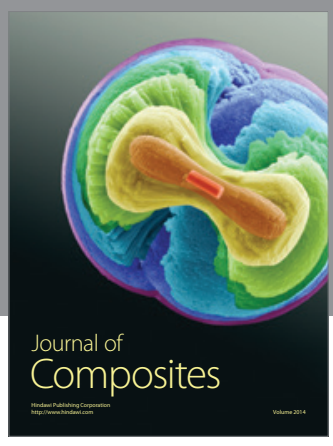
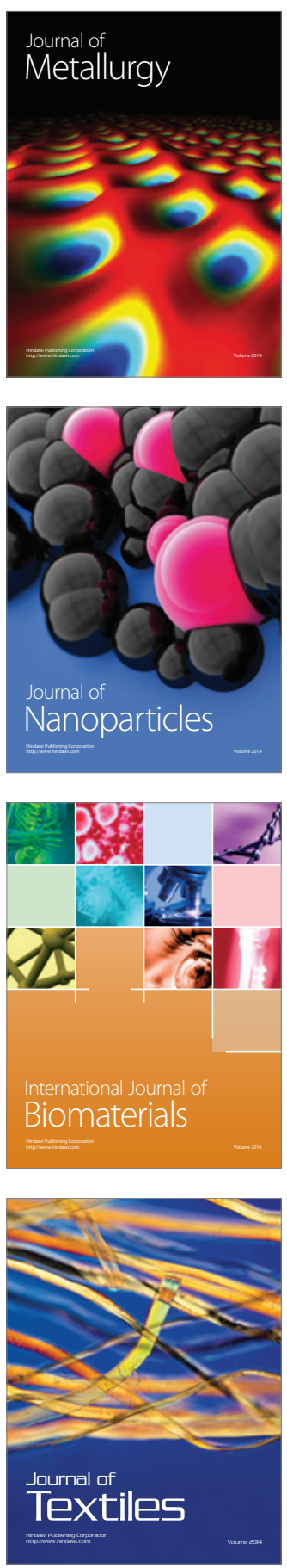